\title{
An Approach to Minimize Very High Density Salt and Pepper Noise through Trimmed Global Mean
}

\author{
T. Veerakumar \\ Assistant Professor \\ Department of ECE \\ PSG College of Technology \\ Coimbatore, India.
}

\author{
S. Esakkirajan \\ Assistant Professor (SG) \\ Department of I\&CE \\ PSG College of Technology \\ Coimbatore, India.
}

\author{
Ila Vennila \\ Associate Professor \\ Department of EEE \\ PSG College of Technology \\ Coimbatore, India.
}

\begin{abstract}
In this paper, we are proposing an approach to minimize very high density salt and pepper noise through trimmed global mean. The Modified decision based unsymmetrical trimmed median filter tries to remove high density salt and pepper noise by taking the mean value of the elements within the processing window. This algorithm fails if all the elements within the processing window is either ' 0 ' or ' 255 '. In our approach, if all the elements within the window are ' 0 ' or ' 255 ' then the noisy pixel is replaced by the trimmed global mean. The proposed algorithm exhibits better image quality than the median filter, adaptive median filter, decision based median filter and modified decision based unsymmetrical trimmed median filter. The proposed algorithm is tested for different grayscale and color images and it gives better peak signal to noise ratio and image enhancement factor.
\end{abstract}

\section{Keywords}

Impulse noise, Median filter, Trimmed Median Filter, Trimmed Global Mean

\section{INTRODUCTION}

Images are frequently corrupted by impulse noise, which is owing to transmission errors, faulty memory locations or timing errors in analog to digital conversion. The two types of impulse noise are random valued noise and salt and pepper noise. This paper concentrates on to remove the salt and pepper noise in an image. Generally, non linear filtering techniques are used in the removal of salt and pepper noise. The standard median filter is one of the most popular nonlinear filter used to remove the impulse noise due to its good

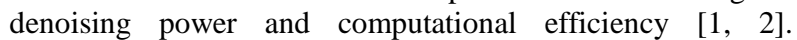
However, the major drawback of standard median filter is that the filter is effective at low noise densities. When the noise density is greater than $50 \%$ the edge details of the original image will not be preserved by median filter. Adaptive median filter (AMF) [3] performs well at low noise densities, at high noise densities the window size has to be increased which may lead to blurring of the image details.

In Decision Based Algorithm (DBA) [4] or switching median filter [5] the decision is based on a threshold value. The major drawback of this method is that defining threshold is difficult [6,7]. Modified Decision based Unsymmetric Trimmed Median Filter (MDBUTMF)[8] performs well at low and high noise densities. At high noise densities, if the selected window contains all 0's or 255's or both then the processing pixel is replaced by mean value of the selected window. The algorithm fails if all the elements within the window are ' 0 ' or ' 255 ' then the mean value will be ' 0 ' or ' 255 ' hence noisy pixel will not be efficiently processed. The proposed algorithm removes this drawback at high noise densities and gives better Peak Signal to Noise Ratio (PSNR) and Image
Enhancement Factor (IEF) values than the existing algorithms.

The rest of the paper is organized as follows. The proposed algorithm is described in Section 2. Section 3 illustrates the proposed algorithm with an example. Experimental results with different test images are given in Section 4. Finally conclusions are drawn in Section 5.

\section{PROPOSED ALGORITHM}

The proposed algorithm starts with the detection of impulse noise in an image. If the processing pixel is greater than 0 and lesser than 255, then the pixel is noise free pixel. Hence the processing pixel is unaltered. If the processing pixel is either 0 or 255 , then the processing pixel is noisy. The noisy pixel is processed by proposed algorithm, which is summarized below:

Step 1: Select 2D window of size $3 \times 3$. Assume that the pixel being processed is $P_{x, y}$.

Step 2: The pixel $\mathrm{P}_{\mathrm{x}, \mathrm{y}}$ is an uncorrupted pixel if $0<\mathrm{P}_{\mathrm{x}, \mathrm{y}}<255$.

Step 3: If the pixel $P_{x, y}=0$ or $P_{x, y}=255$ then the pixel $P_{x, y}$ is a corrupted pixel. The corrupted pixel is processed as follows:

Step 3a: If the selected $3 \times 3$ window contains some of the noise free pixels. Then remove all noisy pixel like 0's and 255 's in a selected window and find the median value of the remaining pixels. (i.e $\mathrm{P}_{\mathrm{x}, \mathrm{y}}$ is replaced by median value).

Step 3b: If the selected window contains all the elements as 0 's and 255 's. Then $P_{x, y}$ is replaced by trimmed global mean.

Step 3c: If the selected window contains all the elements as only 0 's or only 255 's. Then also the processing pixel is replaced by trimmed global mean. The calculation of trimmed global mean is illustrated in Fig. 1.

Step 4: Repeat steps 1 to 3 until all the processing pixel is completed for the entire image.

\subsection{Computation of Trimmed Global Mean}

Let $f(x, y)$ represent a noisy image, the processing pixel is marked as yellow color as shown in Fig. 1. The processing pixel takes a value of 255 which is noisy. It is also possible to observe that all the elements within the processing window take a gray value of 255 . The mean value of the selected window is 255 . This reveals the fact that noise impact is not minimized. In such cases, it would be better to replace the processing pixel by trimmed global mean value of the noise free pixel in the image. The method to obtain trimmed global mean is summarized below:

(a) The image $g(x, y)$ is obtained from $f(x, y)$ by removing all the noisy pixel which is shown in figure 1(b). 


\begin{tabular}{|c|c|c|c|c|c|c|}
\hline 255 & 0 & 128 & 120 & 255 & 115 & 255 \\
\hline 128 & 150 & 156 & 255 & 112 & 0 & 255 \\
\hline 0 & 125 & 255 & 255 & 255 & 255 & 0 \\
\hline 255 & 0 & 255 & 255 & 255 & 110 & 255 \\
\hline 120 & 255 & 255 & 255 & 255 & 255 & 0 \\
\hline 10 & 255 & 35 & 255 & 0 & 20 & 255 \\
\hline 255 & 50 & 0 & 115 & 255 & 255 & 0 \\
\hline
\end{tabular}

(a) Noisy Image $(f(x, y))$

\begin{tabular}{|l|l|l|l|l|l|l|}
\hline & & 128 & 120 & & 115 & \\
\hline 128 & 150 & 156 & & 112 & & \\
\hline & 125 & & & & & \\
\hline & & & & & 110 & \\
\hline 120 & & & & & & \\
\hline 10 & & 35 & & & 20 & \\
\hline & 50 & & 115 & & & \\
\hline
\end{tabular}

(b) Noise free Image $(\mathrm{g}(\mathrm{x}, \mathrm{y}))$

\begin{tabular}{|l|l|l|l|l|l|l|}
\hline & & 128 & 120 & & 115 & \\
\hline 128 & 150 & 156 & & 112 & & \\
\hline & 125 & & & & & \\
\hline & & & 100 & & 110 & \\
\hline 120 & & & & & & \\
\hline 10 & & 25 & & & 18 & \\
\hline & 50 & & 15 & & & \\
\hline
\end{tabular}

(c) Denoised pixel

Fig 1: Computation of Trimmed Global Mean value.

(b) The trimmed global mean $(\mathrm{M})$ of the noise free image $g(x, y)$ is calculated as

$$
M=\frac{1}{N} \sum_{i \in N} \hat{g}(i)
$$

where $\mathrm{N}$ is the number of noise free pixel in an image and $\hat{\mathrm{g}}$ is the noise free element in an image.

(c) Replace the noisy pixel by the trimmed global mean value. In this illustration, the trimmed global mean value is 100 . Hence the noisy pixel value ' 255 ' is replaced by 100 .

\section{ILLUSTRATION OF PROPOSED ALGORITHM}

Every pixels in an image is checked for the presence of salt and pepper noise. Different cases are illustrated in this section.

Case i): If the processing pixel $\left(\mathrm{P}_{\mathrm{x}, \mathrm{y}}\right)$ is in between 0's and 255 's, then the pixel is noise free. Hence, that pixel is unaltered which is illustrated in Fig. 2 (a).

Case ii): If the processing pixel value is 0 or 255 , then the pixel is noisy. Hence, select a $3 \times 3$ window with the processing pixel as the centre element and check all the neighborhood pixels as whether it is 0 or 255 . If some of the pixels within the processing window are noise free pixels, then trimmed median value is computed and is used to replace the corrupted pixel which is shown in Fig. 2 (b). In this case,

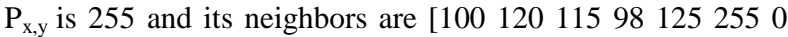
255]. Eliminate the noisy pixels (i.e [100 12011598125255 $\theta$ 255]). Finally, noise free pixels are [llo0 12011598 125]. Therefore, the median value of the noise free pixels is 115 ([98 100115120125$]$ ). The noisy processing pixel 255 is replaced by noise free pixel 115 .

Case iii): If the pixels within the processing window is a combination of only 0's and 255's (i.e [ $\begin{array}{lllll}0 & 0 & 2552550255\end{array}$ $255255]$ ), then the noisy pixel is replaced by the trimmed global mean value which is shown in Fig. 2 (c).

Case iv): If all the pixels within the processing window is either only 0's or only 255's then also the processing pixel is replaced by trimmed global mean value (M), which is illustrated in Fig. 2(d).

\section{RESULTS AND DISCUSSION}

In this section, simulations are carried out on the $256 \times 256$ gray scale Lena and Barbara images to verify the performance of various denoising methods, including MF, AMF, DBA, MDBA, PSMF [9], MDBUTMF and our method. The test images are corrupted by the noise with a wide range of noise densities from 10 to $90 \%$. The Peak Signal to Noise Ratio (PSNR) and Image Enhancement Factor (IEF) are used to evaluate the quantitative quality of the denoised images. The PSNR and IEF are calculated by the equation (2) and (4) respectively.

$$
\begin{array}{r}
P S N R \text { in } d B=20 \log _{10}\left(\frac{255}{M S E}\right) \\
M S E=\frac{1}{M N} \sum_{x} \sum_{y}(I(x, y)-\hat{I}(x, y))^{2}
\end{array}
$$




\begin{tabular}{|l|l|l|}
\hline 100 & 120 & 115 \\
\hline 98 & 105 & 125 \\
\hline 255 & 0 & 255 \\
\hline
\end{tabular}$\quad$\begin{tabular}{|l|l|l|l|}
\hline 100 & 120 & 115 \\
\hline 98 & 105 & 125 \\
\hline 255 & 0 & 255 \\
\hline
\end{tabular}

(a) Case (i)

\begin{tabular}{|c|c|c|c|c|c|c|c|c|c|c|c|c|c|c|}
\hline 100 & 120 & 115 & \multirow{3}{*}{ 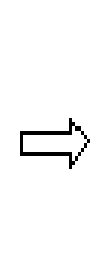 } & 100 & 10 & 115 & \multirow{3}{*}{ 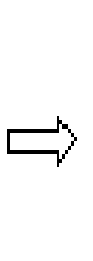 } & 1010 & 20 & 115 & \multirow{3}{*}{$\hookrightarrow$} & 100 & 120 & 115 \\
\hline 98 & 255 & 25 & & $\phi$ & 25 & 125 & & $\phi$ & & 125 & & 98 & $11 \%$ & 25 \\
\hline 255 & 0 & 255 & & 255 & 0 & 255 & & & & & & 25 & 0 & 25 \\
\hline
\end{tabular}

(b) Case (ii)

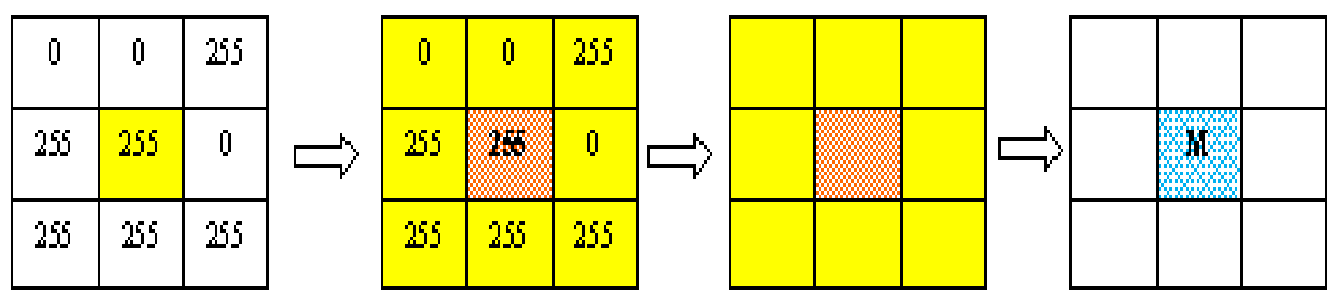

(c) Case (iii)

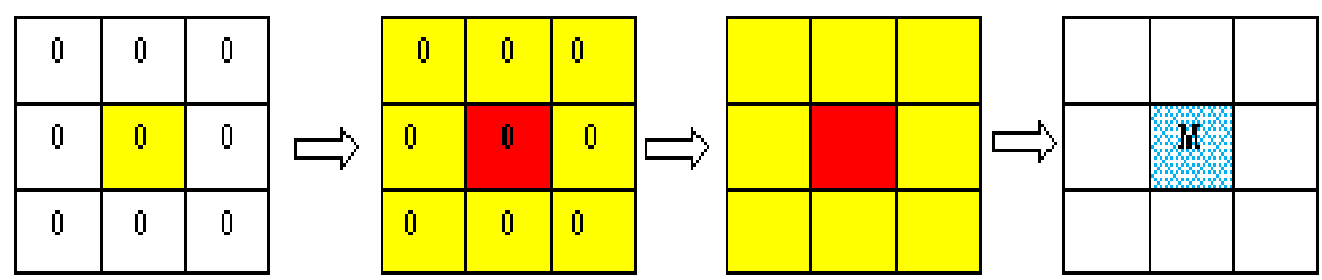

(d) Case (iv)

Fig 2: Illustration of Proposed algorithm.

$$
I E F=\frac{\sum_{x} \sum_{y}(\eta(x, y)-I(x, y))^{2}}{\sum_{x} \sum_{y}(\hat{I}(x, y)-I(x, y))^{2}}
$$

where MSE stands for mean square error, MN is the size of the image, I represents the original image, $\hat{I}$ represents the denoised image and $\eta$ denotes the noisy image.

Figure 3, shows the results of different methods in denoising a $75 \%$ and $90 \%$ salt and pepper noise corrupted Lena image. From the figure, it is possible to observe that the proposed algorithm performs significantly better than the existing methods. The PSNR values of the proposed algorithm are compared against the existing methods by varying the noise densities from 10 to $90 \%$ and is shown in Table 1 . From the table, it is evident that the performance of the proposed algorithm is better than the existing algorithms at high noise densities. The PSNR results of the proposed algorithm is compared against the existing algorithms and the plot is shown in figure 4. Apparently, the performance of the proposed method is marginally better than the existing methods at high noise densities.

The IEF values of the proposed algorithm is compared against the existing methods by varying the noise densities from 10 to $90 \%$ and the results are shown in table 2. From the table, it is possible to conclude that the proposed algorithm outperforms the existing algorithms at high noise densities. The proposed method is also tested for color Barbara image that are corrupted with impulse noise. In figure 5, the first column represents the denoised image using $\mathrm{MF}$ at 70 and $85 \%$ noise densities. Subsequent columns represent the denoised image for AMF, DBA, MDBA, MDBUTMF and proposed method. From the figure, it is possible to observe that the quality of the denoised image using proposed method is better than the quality of the denoised image using existing algorithms. 
Table 1. PSNR Values for Various Algorithms for Lena Image at Different Noise Density

\begin{tabular}{|c|c|c|c|c|c|c|}
\hline \multirow{2}{*}{$\begin{array}{c}\text { Noise Variance } \\
\text { in \% }\end{array}$} & \multicolumn{7}{|c|}{ PSNR in dB } \\
\cline { 2 - 6 } & MF & AMF & DBA & MDBA & MDBUTMF & Proposed \\
\hline 10 & 28.71 & 21.99 & 36.80 & 36.80 & 38.47 & 37.99 \\
\hline 20 & 25.49 & 21.90 & 32.65 & 32.65 & 34.29 & 33.90 \\
\hline 30 & 22.08 & 21.85 & 30.74 & 30.74 & 32.30 & 32.12 \\
\hline 40 & 18.11 & 21.58 & 28.51 & 28.68 & 30.34 & 30.22 \\
\hline 50 & 14.90 & 20.60 & 26.54 & 26.56 & 28.44 & 28.55 \\
\hline 60 & 12.29 & 18.35 & 24.29 & 24.51 & 26.43 & 26.54 \\
\hline 70 & 9.84 & 14.71 & 22.07 & 22.19 & 24.28 & 24.45 \\
\hline 80 & 8.02 & 11.25 & 20.40 & 20.43 & 21.70 & 22.01 \\
\hline 90 & 6.66 & 8.22 & 17.22 & 17.50 & 17.94 & 18.25 \\
\hline
\end{tabular}

Table 2. IEF Values for Various Algorithms for Lena Image at Different Noise Density

\begin{tabular}{|c|c|c|c|c|c|c|}
\hline \multirow{2}{*}{$\begin{array}{c}\text { Noise Variance } \\
\text { in \% }\end{array}$} & \multicolumn{7}{|c|}{ PSNR in dB } \\
\cline { 2 - 7 } & MF & AMF & DBA & MDBA & MDBUTMF & Proposed \\
\hline 10 & 20.67 & 4.38 & 133.14 & 133.14 & 195.36 & 174.89 \\
\hline 20 & 20.04 & 8.78 & 104.27 & 104.12 & 151.97 & 138.93 \\
\hline 30 & 13.70 & 12.96 & 100.51 & 100.54 & 144.06 & 138.08 \\
\hline 40 & 7.39 & 16.46 & 81.21 & 84.34 & 123.84 & 120.31 \\
\hline 50 & 4.42 & 16.40 & 64.36 & 64.71 & 99.87 & 102.21 \\
\hline 60 & 2.88 & 11.62 & 45.61 & 47.96 & 74.59 & 76.53 \\
\hline 70 & 1.94 & 5.95 & 32.37 & 33.28 & 53.89 & 56.05 \\
\hline 80 & 1.45 & 3.06 & 25.12 & 25.32 & 33.91 & 36.41 \\
\hline 90 & 1.19 & 1.70 & 13.52 & 14.39 & 15.88 & 17.07 \\
\hline
\end{tabular}

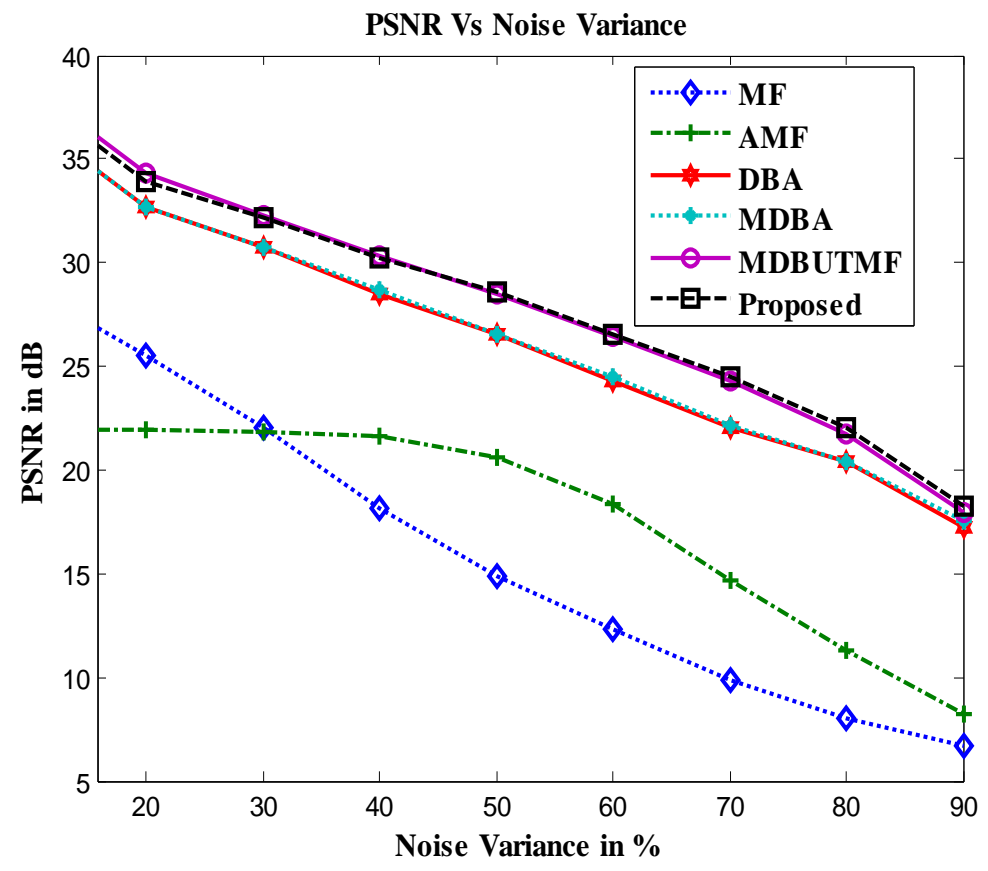

Fig 4: Comparison of denoised results in PSNR for Lena with various noise densities.

\section{CONCLUSION}

In this paper, a new algorithm is proposed for the removal of very high density salt and pepper noise in an image. The proposed algorithm eliminates the drawback of MDBUTMF when all the elements within the processing window is either 0 or 255 or both. If all the pixels within the processing window happens to be 0 or 255 or both, the size of the window can be increased which leads to adaptive algorithm which is computationally intensive. On the other hand, the proposed algorithm replaces the corrupted pixel by trimmed mean value. The computation of trimmed global mean is noniterative, simple and easy to implement. Simulation results demonstrate the validity of the proposed method. 


\section{REFERENCES}

[1] Astola, J. and Kuosmanen, P. 1997. Fundamentals of Nonlinear Digital Filtering.Boca Raton, FL: CRC.

[2] Huang, T. S., Yang, G. J., and Tang, G. Y. 1979. Fast two-dimensional median filtering algorithm, IEEE Trans. Acoustics, Speech, Signal Process. 1 (Jan. 1979), 13-18.

[3] Hwang, H., and Hadded, R. A. 1995. Adaptive median filter: New algorithms and results, IEEE Trans. Image Process. 4 (Apr. 1995), 499-502.

[4] Srinivasan K. S., and Ebenezer, D. 2007. A new fast and efficient decision based algorithm for removal of high density impulse noise, IEEE Signal Process. Lett. 14 (Mar. 2007), 189-192.

[5] Ng, P. E., and Ma, K. K. 2006. A switching median filter with boundary discriminative noise detection for extremely corrupted images, IEEE Trans. Image Process., 15 (Jun. 2006), 1506-1516.
[6] Luo, W. 2006. An efficient detail-preserving approach for removing impulse noise in images, IEEE Signal Process. Lett. 13 (Jul. 2006), 413-416.

[7] Chen, P. Y., and Lien, C. Y. 2008. An Efficient EdgePreserving Algorithm for Removal of Salt-and-Pepper Noise, IEEE Signal Processing Letters. 15(2008), 833836.

[8] Esakkirajan, S., Veerakumar, T., Subramanyam, A.N., and PremChand, C. H. 2011. Removal of High Density Salt and Pepper Noise Through Modified Decision Based Unsymmetric Trimmed Median Filter, IEEE Signal Process. Lett. 18 (May 2011), 287-290.

[9] Wang, Z., and Zhang, D. 1999. Progressive switching median filter for the removal of impulse noise from highly corrupted images, IEEE Trans. Circ. Syst.-II: Analog Digit. Signal Process. 46 (Jan. 1999), 78-80.

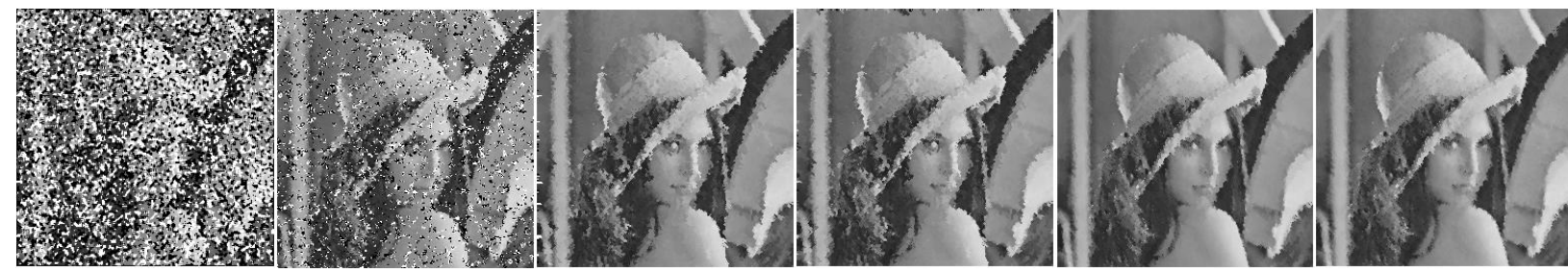

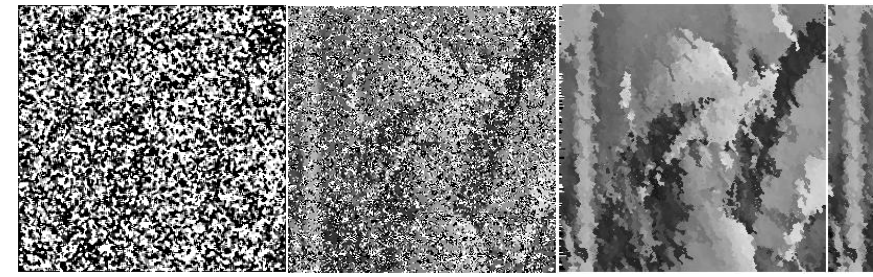

MF

\begin{abstract}
DBA
\end{abstract}

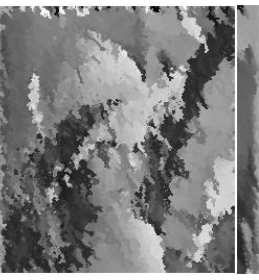

MDBA

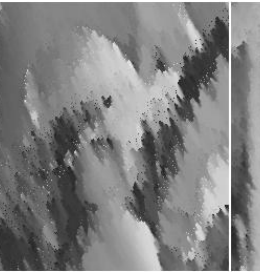

MDBUTMF

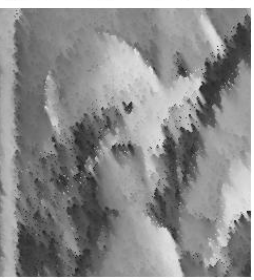

PROPOSED

Fig 3: Restoration results of different method for Lena image corrupted with $75 \%$ (Row 1) and $90 \%$ (Row 2) noise density salt and pepper noise.
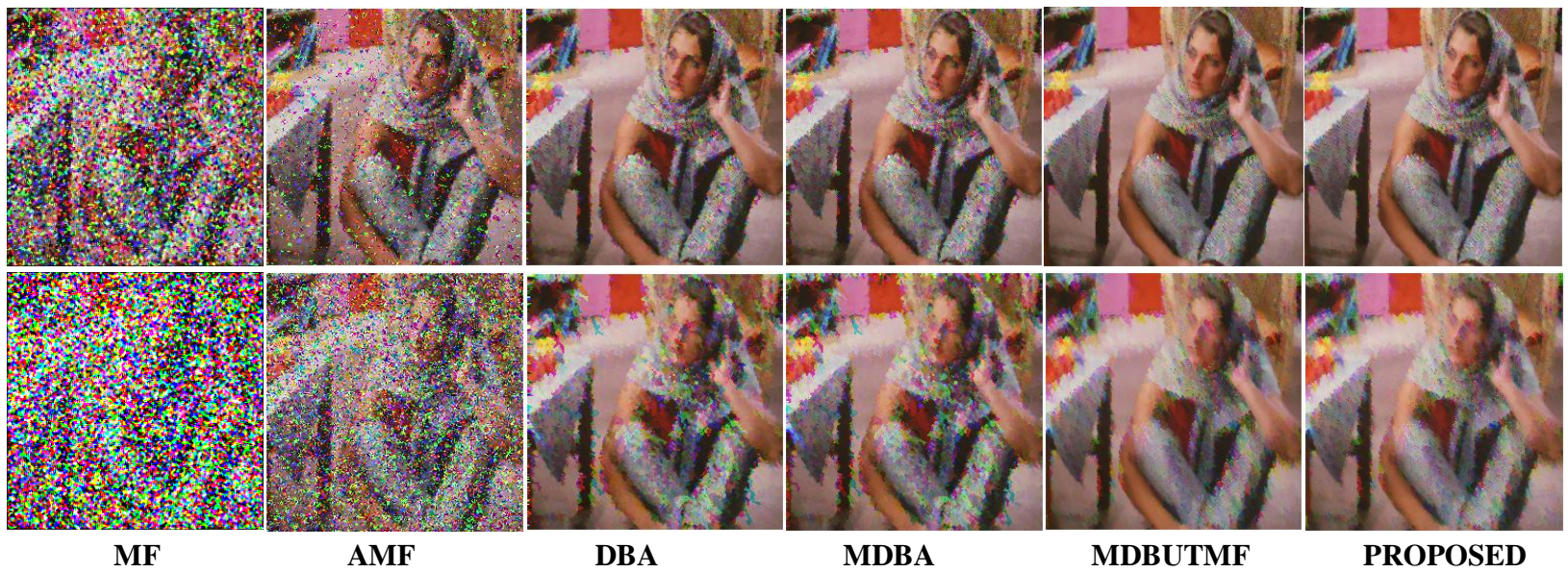

Fig 5: Restoration results of different method for color Barbara image corrupted with 70\% (Row 1) and 85\% (Row 2) noise density salt and pepper noise. 